Ciência Florestal, Santa Maria, v. 2, n. 1, p. 161-171, 1992

ISSN 0103-9954

\title{
EFEITO DA MASSA ESPECÍFICA NAS PROPRIEDADES DE FLEXÃO ESTÁTICA EM CONPENSADOS DE Pinus elliottii Engelm
}

\section{EFECT OF THE SPECIFIC GRAVITY ON STATIC BENDING PROPERTIES IN PLYWOOD OF Pinus elliottii Engelm}

\author{
Hernando Alfonso Lara Palma ${ }^{1}$
}

\section{RESUMO}

O presente trabalho teve por objetivo a determinação de relações entre as propriedades de resistência e rigidez à flexão estática e a massa especifica de painéis compensados de 5 lâminas feitos de madeira de Pinus elliottii Engelm, proveniente de plantios de 30 anos de idade. Foram estudadas várias relações lineares simples, múltiplas e polinomiais, envolvendo o módulo de ruptura (MOR), o módulo de elasticidade (MOE), a tensão no limite proporcional (TLP) e a massa específica (Me) dos compensados. A massa específica mostrou não ser indicador muito preciso das propriedades de resistência e rigidez dos painéis. As propriedades dos compensados à flexão estática, em termos de MOE, MOR e TLP mostraram baixos coeficientes de correlação e determinação ajustados por regressão linear simples, indicando pouca dependência de massa específica. O módulo de ruptura à flexão nas direções principais (x e Y), teve uma forte dependência do módulo de elasticidade e de tensão no limite proporcional. Os coeficientes de correlação dos melhores modelos de regressão nas duas direções variaram de 0,85 a 0,91 , Histogramas de frequência da massa específica dos compensados mostraram uma distribuição unimodal homogênea, enquanto que o MOE e o MOR apresentaram uma distribuição unimodal com assimetria positiva.

Palavras-chave: Compensados, lâminas, flexão estática em compensados, Pinus elliottii.

\section{ABSTRACT}

The objetive of this work was to determine the relation between the rigidity and strength properties in static bending and specific gravity of plywood manufactured

1 Engenheiro em Indústrias da Madeira, Dr., Professor do Departamento de Ciências Florestais, Faculdade de Ciências Agrárias, Universidade Estadual Paulista, CEP: 18603-970, Botucatu - SP. 
from five veneers of Pinus elliottii Engelm from 30 year-old plantations. Linear simples, multiple and polynomial relations involving the modulus of rupture (MOR), modulus of elasticity (MOE), stress at proportional limit (TLP) and specific gravity (Me) of the plywood were studied. The specific gravity did not prove to be a very exact indicator of the rigidity and strength properties of the plywood. The MOE, MOR and TLP in static bending of the plywood showed low coefficients of correlations and determination adjusted for simple linear regression, indicating a little dependence of the specific gravity, the modulus of rupture in static bending on the principal directions (X and $\mathrm{Y}$ ) had a strong dependence of the modulus of elasticity and stress at proportional limit. The coefficients of correlations of the best models of regression in the two directions varied from 0.85 to 0,91 . The frequency histograms of the specific gravity of plywood showed a unimodal homogeneous distribution, while MOE and MOR showed a unimodal distribution with positive asymmetric skewness.

Key words: Plywoods, veneer, static bending in plywood, Pinus elliottii.

\section{INTRODUÇÃO}

O conhecimento das propriedades de rigidez e de resistência da madeira compensada é indispensável para diversas soluções construtivas, a fim de permitir uma avaliação da capacidade desta estrutura no seu limite máximo.

Com o aumento das restrições ambientais e do distanciamento das reservas naturais das espécies tradicionais, utilizadas na manufatura de compensados no país, o abastecimento passou a ser prejudicado. Para contornar este problema, uma das opções foi a utilização de madeiras (laminadas) de reflorestamentos, principalmente de espécies do gênero Pinus.

Do ponto de vista tecnológico, a maioria dos produtos laminados de madeira refletem de certa maneira as propriedades das espécies das quais foram manufaturados. Portanto, faz-se necessário quantificar as mudanças que possam ocorrer nas propriedades dessa nova matéria-prima e que podem afetar as propriedades físicas e mecânicas dos compensados.

\section{REVISÃO DE LITERATURA}

A madeira é um produto orgânico sendo usada, principalmente, em seu estado natural, constituindo-se de camadas concêntricas de crescimento. Produzida por árvores que crescem sob condições ecológicas variáveis que, naturalmente, 
afetam suas taxas de crescimento, forma, estrutura e resistência, variáveis estas que tornam difícil atribuir valores de esforços de trabalho com o mesmo grau de precisão que outros materiais.

As diferenças no arranjo dos tecidos, dimensões e espessuras da parede do lúmen celular determinam valores próprios da massa específica para cada espécie de madeira. Salienta-se que a resistência da madeira está estreitamente relacionada com a sua massa específica.

Segundo Lutz (1971), as propriedades físicas e mecânicas da madeira de maior interesse para a produção de lâminas para compensados, são: a massa específica, permeabilidade, contração, módulo de elasticidade longitudinal, módulo de ruptura, cisalhamento longitudinal e perpendicular das fibras, tração perpendicular e dureza. A influência de tratos silviculturais aplicados intensamente em reflorestamentos, como por exemplo, podas e ciclos menores de rotação afetam a qualidade da madeira (BUNN, 1981). Assim, mudanças em variáveis, tais como massa específica, tamanho e distribuição de nós e largura dos anéis de crescimento é esperada, portanto, as propriedades das lâminas obtidas desta matéria-prima dependem destas mudanças.

De acordo com Bier (1983b), é essencial determinar como estas mudanças, em particular, a massa específica e o tamanho de nós podem afetar as propriedades mecânicas em compensados de Pinus, e assim predizer adequadamente o seu desempenho estrutural. Algumas mudanças na magnitude destas propriedades afetam diretamente as tensões básicas de trabalho.

Lutz (1964) observou a necessidade de se considerar a grande variação existente entre os lenhos tardio e inicial de coníferas na qualidade das lâminas destinadas à confecção de compensados.

A resistência final de um painel compensado depende fortemente da distribuição dos lenhos tardio e inicial nas lâminas. As propriedades físicas e mecânicas nos dois tipos de lenho, em madeira de Pinus de rápido crescimento, são variáveis, sendo que, a massa específica, segundo diversos autores, varia em torno de $0,30 \mathrm{~g} / \mathrm{cm}^{3}$ para o lenho inicial e 0,65 a $0,90 \mathrm{~g} / \mathrm{cm}^{3}$ para o lenho tardio, com uma diferença em resistência de 3 a 5 vezes maior neste último (LUTZ, 1964; CAMARGO, 1987; MUÑIZ, 1993 e KOCH, 1965).

Em geral, árvores de rápido crescimento de espécies do gênero Pinus, apresentam os primeiros anéis de crescimento, a partir da medula, bastante largos e a faixa de lenho tardio estreita e os anéis mais próximos da casca mais estreitos e uma maior percentagem de lenho tardio. A largura média dos anéis de crescimento na madeira de Pinus de rápido crescimento pode variar de 3 a $8 \mathrm{~mm}$ aproximadamente e de 50 a 70\% a proporção do lenho inicial no anel (MUÑIZ, 
1993).

Isto é relevante do ponto de vista da laminação, quando se considera que o corte da madeira é feito principalmente, na direção tangencial da tora e em parte na direção radial, devido à conicidade que apresentam as árvores, obtendo-se assim lâminas com distribuição de ambos os lenhos através da espessura. Portanto, ao se laminarem madeiras de coníferas de rápido crescimento em espessuras mais finas, corre-se o risco de obter lâminas com grande influência de lenho inicial, o que implicaria em uma menor resistência do painel. Este fato é menos importante em lâminas produzidas de madeiras de crescimento lento (nativas), nas quais essa distribuição fica diminuída, pela pequena largura dos anéis de crescimento.

Segundo Lutz (1964), a grande diferença nas propriedades nos dois lenhos de crescimento é uma característica importante na confecção de compensados para uso estrutural, pois, o lenho tardio distribuído nas lâminas fornece maior resistência ao painel.

De acordo com o autor acima, a maioria das lâminas obtidas de madeira que tem anéis de crescimento estreitos possui uma boa distribuição de lenho tardio e, consequentemente, melhor resistência. Ao contrário, nas madeiras que possuem anéis de crescimento largos, é necessário que o lenho tardio represente no mínimo $50 \%$ do anel anual, para se obterem lâminas com propriedades semelhantes às anteriores. Por outro lado, lâminas produzidas com ao menos dois anéis de crescimento ou mais através da espessura, reduzem durante o processo de corte (desenrolamento) a Profundidade das fendas de laminação e a separação do lenho inicial produzida pela faca e pela flexão contrária das lâminas na saída do torno, o que implica na produção de lâminas com baixas propriedades de flexão.

Bier (1983a) apresentou um estudo sobre as relações entre as propriedades de resistência e rigidez à flexão do compensado de Pinus radiada, com variáveis tais como: taxa de nós das lâminas, massa específica das lâminas e massa específica do painel. Os resultados mostraram pouca dependência entre o módulo de ruptura com a massa específica do painel ao contrário do módulo de elasticidade. A resistência mostrou-se fortemente influenciada pela taxa de nós, e a massa específica do compensado apresentou pouca correlação com a massa específica das lâminas componentes. O conhecimento desta relação é importante para manter a construção balanceada (BODIG \& JAYNE 1993).

Bier (1984) apresentou, em outro estudo, a influência da massa específica nas propriedades de rigidez e de resistência de compensados estruturais de Pinus. Os resultados obtidos mostraram que apesar da grande dispersão nos dados, evidenciou-se sempre um aumento nas propriedades de rigidez e de resistência à flexão paralela às fibras com o aumento da massa específica do painel. 
Outro resultado relevante do estudo acima foi que para uma distribuição de frequências da massa específica quase homogênea do compensado, existe, sempre uma distribuição muito irregular nas frequências das propriedades de rigidez e resistência do painel. Segundo esse autor, as razões deste comportamento podem ser atribuídas à grande diferença de massa específicas entre as lâminas componentes do painel e também às características da última lâmina submetida à tensão, que governa a resistência à flexão no corpo de prova e que, no caso de possuir uma maior percentagem de lenho inicial, resultará em baixa resistência do compensado para uma massa específica aparentemente alta.

\section{MATERIAL E MÉTODOS}

\section{Amostragem, coleta de material e estudo das propriedades físicas e mecânicas dos compensados}

A madeira utilizada neste trabalho foi obtida de árvores de Pinus elliottii Engelm provenientes de plantios de 30 anos de idade, localizados na Floresta Nacional de Irati, Paraná.

A amostragem das árvores foi seletiva, escolhendo-se entre as de maior diâmetro e com fuste cilíndrico.

Após a seleção foram obtidas quatro árvores e de cada árvore foram coletados dois toretes de $1,20 \mathrm{~m}$ de comprimento, tomados nos primeiros oito metros, perfazendo um total de oito toretes.

De cada torete foram feitos dez compensados de 5 lâminas de $2 \mathrm{~mm}$ cada, com dimensões nominais de 600 x 600 x $10 \mathrm{~mm}$, perfazendo 20 compensados em cada árvore e um total de 80 para a espécie.

As variáveis do processo de fabricação dos painéis foram escolhidas levando-se em consideração as recomendações do fabricante do adesivo fenolformaldeído utilizado. Assim, as variáveis foram fixas para todos os painéis como segue: temperatura de prensagem $130^{\circ} \mathrm{C}$, pressão de prensagem $9 \mathrm{~kg} / \mathrm{cm}^{3}, 360 \mathrm{~g} /$ $\mathrm{m}^{2}$ de adesivo por linha dupla de colagem e $6 \%$ de teor de umidade das lâminas.

Na avaliação dos painéis, as determinações principais foram a resistência à flexão estática paralela e perpendicular à grã da face e a massa específica aparente. Os corpos de prova foram confeccionados segundo as prescrições das normas recomendadas pela DIN—52371 para compensados.

Para o teste de flexão estática foram retirados de cada chapa dez corpos de prova, cinco para paralela e cinco para flexão perpendicular às fibras, perfazendo um total de 800 amostras. Para o cálculo da massa específica foi retirada uma 
amostra perto da região da fratura do corpo de prova de flexão, perfazendo também um total de 800 amostras para a espécie.

Todos os ensaios foram feitos com as amostras tendo um teor de umidade de aproximadamente $12 \%, 20 \% \pm 2{ }^{\circ} \mathrm{C}$ e $65 \pm 2{ }^{\circ} \mathrm{C}$ de UR.

\section{Análise estatística}

Com o objetivo de estabelecer relações entre as propriedades estudadas nos compensados, foram feitas análises de regressão, utilizando-se os seguintes modelos de equações para o ajuste de curvas: modelo linear simples $\left(y=b_{0}+b_{1} x\right)$, modelo linear com duas variáveis $\left(\mathrm{y}=\mathrm{b}_{0}+\mathrm{b}_{1} \mathrm{X}_{\mathrm{I}}+\mathrm{b}_{2} \mathrm{X}_{2}\right)$ e modelo polinomial de 20 grau $\left(\mathrm{y}=\mathrm{b}_{0}+\mathrm{b}_{1 \mathrm{x}}+\mathrm{b}_{2} \mathrm{X}^{2}\right)$.

Foram testadas, como variáveis dependentes, o módulo de elasticidade (MOE), o módulo de ruptura (MOR) e a tensão no limite proporcional (TLP) do compensado, e como variáveis independentes o MOE, o MOR a TLP e a massa específica aparente (Me).

$\mathrm{O}$ ajuste e a precisão das equações foram avaliados pelo coeficiente de determinação correlação (r) e o erro padrão de estimativa $\left(\mathrm{S}_{\mathrm{yx}}\right)$.

\section{RESULTADOS E DISCUSSÃO}

As equações apresentadas na Tabela 1 foram as de melhor ajuste para estimar os parâmetros elásticos e de resistência dos compensados, no sentido paralelo e perpendicular.

As tentativas para se obter um valor mais elevado de $\mathrm{R}$, com a introdução em cada modelo de pelo menos mais um termo linear ou ao quadrado não foram muito significativas, como pode ser evidenciado nas equações 7 a 12 e 19 a 22 .

Observando-se o coeficiente de determinação $\left(\mathrm{R}^{2}\right)$ e o erro padrão da estimativa $\left(\mathrm{S}_{\mathrm{xy}}\right)$, constata-se que as equações que melhor se ajustaram aos dados para o MOR são as originadas do modelo 3, 4, 10 e 12, para o MOE, as equações 7 e 8 e para a TLP, as equações 6 e 11, no caso dos compensados no sentido paralelo. Para os resultados obtidos no sentido perpendicular às fibras, os modelos que melhor se ajustaram aos dados seguiram a mesma tendência apresentada no sentido paralelo, ao igual que os valores de $\mathrm{R}^{2}$.

As Figuras 1 e 2 apresentam para os modelos 1, 2, 5, 3, 4 e 6, respectivamente, os gráficos das equações ajustadas, bem como as tendências dos valores ajustados, para os compensados no sentido paralelo. 
TABELA 1: Equações de regressão das propriedades de flexão estática dos compensados.

TABLE 1: Regression equations of properties of static bending of plywoods.

\begin{tabular}{|c|c|c|c|c|c|c|}
\hline Eq. N. & Modelos e coeficientes & $\mathrm{r}$ & $\mathrm{R}^{2}$ & Syx & $\operatorname{Syx}(\%)$ & $\mathrm{F}$ \\
\hline \multicolumn{7}{|c|}{ a) Para testes paralelos à grã da face ${ }^{1}$} \\
\hline (1) & $\mathrm{MOE}=212560 \mathrm{Me}-8640$ & 0,57 & 0,33 & 21408 & 31,61 & $197,59 * *$ \\
\hline (2) & $\mathrm{MOR}=1546 \mathrm{Me}-279$ & 0,65 & 0,42 & 130 & 21,78 & $1041,10 * *$ \\
\hline (3) & $\mathrm{MOR}=0,0055 \mathrm{MOE}+223$ & 0,85 & 0,72 & 89 & 14,91 & $283,16^{* *}$ \\
\hline (4) & $\mathrm{MOR}=1,16 \mathrm{TLP}+101$ & 0,91 & 0,82 & 72 & 12,06 & $1800,70^{* *}$ \\
\hline (5) & $\mathrm{TLP}=1150 \mathrm{Me}-226$ & 0,62 & 0,38 & 104 & 24,41 & $245,09 * *$ \\
\hline (6) & $\mathrm{TLP}=0,0046 \mathrm{MOE}+112$ & 0,92 & 0,84 & 52 & 12,21 & $2138,00 * *$ \\
\hline (7) & $\begin{array}{c}\mathrm{MOE}=126 \mathrm{MOR}+17426 \mathrm{Me}- \\
17515\end{array}$ & 0,85 & 0,72 & 13757 & 20,31 & $522,62 * *$ \\
\hline (8) & $\begin{array}{c}\mathrm{MOE}=24749+14 \mathrm{MOR}+0,09 \\
\mathrm{MOR}^{2}\end{array}$ & 0,86 & 0,74 & 13408 & 19,80 & $560,71 * *$ \\
\hline (9) & $\begin{array}{c}\mathrm{MOE}=77180-245134 \mathrm{Me}+396791 \\
\mathrm{Me}^{2}\end{array}$ & 0,58 & 0,34 & 21293 & 31,44 & $102,53 * *$ \\
\hline$(10)$ & $\mathrm{MOR}=556 \mathrm{Me}+0,0047 \mathrm{MOE}-33$ & 0,87 & 0,76 & 84 & 14,07 & $626,23 * *$ \\
\hline (11) & $\mathrm{TLP}=247 \mathrm{Me}+0,0043 \mathrm{MOE}-1,8$ & 0,92 & 0,85 & 51 & 11,97 & $1168,40 * *$ \\
\hline$(12)$ & $\mathrm{MOR}=333 \mathrm{Me}+1,05 \mathrm{TLP}-41$ & 0,91 & 0,83 & 70 & 11,73 & $975,70 * *$ \\
\hline \multicolumn{7}{|c|}{ b) Para testes perpendiculares à grã da face ${ }^{1}$} \\
\hline$(13)$ & $\mathrm{MOE}=60135 \mathrm{Me}-11669$ & 0,58 & 0,34 & 5945 & 27,06 & $173,08 * *$ \\
\hline (14) & $\mathrm{MOR}=766 \mathrm{Me}-130$ & 0,64 & 0,41 & 65 & 21,76 & $238,51^{* *}$ \\
\hline$(15)$ & $\mathrm{MOR}=0,01 \mathrm{MOE}+76$ & 0,88 & 0,77 & 40 & 13,39 & $1169,80 * *$ \\
\hline$(16)$ & $\mathrm{MOR}=1,30 \mathrm{TLP}+64$ & 0,89 & 0,80 & 38 & 12,72 & $1370,90 * *$ \\
\hline (17) & $\mathrm{TLP}=0,007 \mathrm{MOE}+028$ & 0,87 & 0,76 & 28 & 15,52 & $1100,40 * *$ \\
\hline$(18)$ & $\mathrm{TLP}=506 \mathrm{Me}-102$ & 0,62 & 0,38 & 46 & 25,50 & $207,80 * *$ \\
\hline (19) & $\mathrm{MOE}=2769 \mathrm{Me}+75 \mathrm{MOR}-1948$ & 0,88 & 0,77 & 3470 & 15,59 & $584,61 * *$ \\
\hline$(20)$ & $\mathrm{MOR}=236 \mathrm{Me}+0,009 \mathrm{MOE}-27$ & 0,89 & 0,80 & 38 & 12,72 & $680,65 * *$ \\
\hline$(21)$ & $\mathrm{TLP}=134 \mathrm{Me}+0,006 \mathrm{MOE}-30$ & 0,88 & 0,78 & 27 & 14,97 & $606,69 * *$ \\
\hline$(22)$ & $\mathrm{MOR}=175 \mathrm{Me}+1,17 \mathrm{TLP}-10$ & 0,90 & 0,81 & 36 & 12,05 & $744,45^{* *}$ \\
\hline
\end{tabular}

Em que: ${ }^{1} \mathrm{o}$ número de amostras testadas $(\mathrm{N})$ foi de $400 ; * *$ Significativo em nível de $1 \%$ de probabilidade. 
Com relação ao comportamento dos dados nas equações 1,2 e 5, observase que a massa específica não é um indicador muito preciso do MOE, do MOR e da TLP, comportamento semelhante também no sentido perpendicular. De uma maneira geral, apesar da dispersão dos valores, nota-se que um aumento na massa específica do compensado implica em um aumento do MOE, MOR e TLP.

Com relação ao comportamento dos dados das equações 3, 4, 15 e 16, observase que o módulo de ruptura nas duas direções principais está bem correlacionado ao módulo de elasticidade e à tensão no limite proporcional, melhorando muito pouco com a inclusão da massa específica. Com base nas correlações obtidas das regressões lineares, observaram-se coeficientes de correlação nos dois sentidos de 0,85 a 0,88 para o MOE, 0,85 a 0,91 para o MOR e 0,87 a 0,92 para a TLP. Na Figura 2, observam-se as tendências destes valores ajustados no sentido paralelo.

Além disso, pode-se observar, nos histogramas de distribuição de frequências da Figura 3, que a massa específica dos compensados apresentou uma distribuição unimodal com uma tendência nas classes centrais mais retangular para a maioria dos valores observados, enquanto que as propriedades de resistência e rigidez apresentaram uma distribuição unimodal com assimetria positiva.

Relacionando estes gráficos (Figura 3) com os coeficientes de variação (Tabela 2), observa-se que a variação da massa específica é muito menor que o MOR e MOE, portanto, para valores próximos ou sementes de massas específicas dos painéis, existem valores de resistência e rigidez com amplitudes

TABELA2: Resultados médios de flexão estática e massa específica dos compensados.

TABLE 2: Average results of static flexion and specific mass of plywoods.

\begin{tabular}{|c|c|c|c|c|c|c|c|c|}
\hline \multirow{2}{*}{ Estatística } & \multicolumn{2}{|c|}{$\operatorname{MOE}\left(\mathrm{Kgf} / \mathrm{cm}^{2}\right)$} & \multicolumn{2}{|c|}{$\operatorname{MOR}\left(\mathrm{kgf} / \mathrm{cm}^{2}\right)$} & \multicolumn{2}{|c|}{$\operatorname{TLP}\left(\mathrm{Kgf} / \mathrm{cm}^{2}\right)$} & \multicolumn{2}{|c|}{$\begin{array}{l}\text { Massa Específica } \\
\qquad\left(\mathrm{g} / \mathrm{cm}^{3}\right)\end{array}$} \\
\hline & Paralelo & Perpendicular & Paralelo & Perpendicular & Paralelo & Perpendicular & Paralelo & Perpendicular \\
\hline Media & 67729,64 & 21970,38 & 596,93 & 298,66 & 426,04 & 180,42 & 0,567 & 0,559 \\
\hline S & 26155,43 & 7289,29 & 169,89 & 83,97 & 132,07 & 57,79 & 0,071 & 0,070 \\
\hline C.V. & 38,60 & 33,18 & 28,46 & 28,12 & 31,00 & 32,03 & 12,52 & 12,52 \\
\hline
\end{tabular}



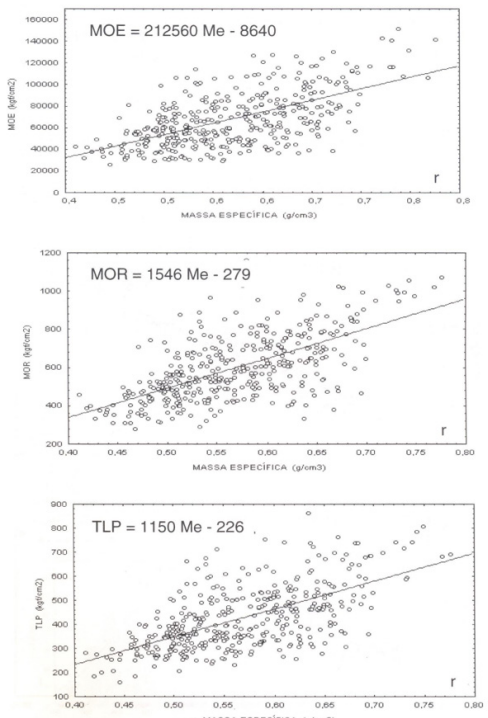

FIGURA 1: Representação gráfica das equações de regressão simples, ajustadas para o MOE, o MOR, a TLP e a massa específica.

FIGURE 1: Graphical representation of simple regression equations, adjusted for MOE, MOR, TLP and specific mass.

\section{CONCLUSÃO}

Com base nos resultados apresentados, para as propriedades dos compensados de Pinus elliottii Engelm, pode-se concluir:

1. As propriedades de resistência e rigidez dos compensados à flexão estática, em termos de módulo de elasticidade, módulo de ruptura e tensão no limite de proporcionalidade, mostraram baixos coeficientes de correlação e determinação ajustados por regressão linear simples (nos dois sentidos principais), indicando pouca dependência da massa específica.

2. A resistência dos compensados, em termos de módulo de ruptura à flexão estática (nos dois sentidos testados), mostrou uma forte dependência do módulo de elasticidade e da tensão no limite de proporcionalidade.

3. Existe uma alta variação na amplitude dos valores das propriedades de resistência e rigidez dos compensados, para uma variação pequena e muito 

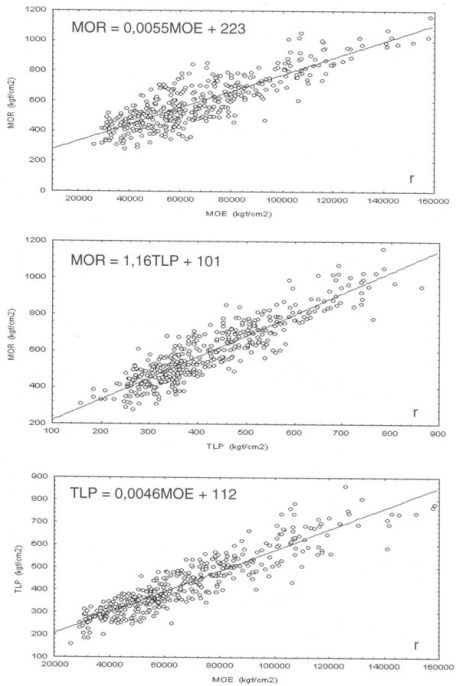

FIGURA 2: Representação gráfica das equações de regressão simples, ajustadas para o MOE e o MOR, o MOR e a TLP e a TLP e o MOE para compensados no sentido paralelo.

FIGURE 2: Graphical representation of the simple regression equations, adjusted for MOE and MOR, MOR and TLP, and TLP and MOE for plywoods in the parallel sense.

semelhante da massa específica dos painéis.

\section{REFERÊNCIAS BIBLIOGRÁFICAS}

BIER, H. Radiata pine plywood - A theorical prediction of the bending properties of structural plywood. New Zealand Florest Service, FRI Bulletin, n. 54, $1983 \mathrm{a}$. BIER, H. Radiata pine plywood - An experimental study of the properties of structural plywood. New Zealand Florest Service, FRI Bulletin, n. 53, 1983b. BIER, H. Radiata pine plywood - The strength properties of small clear specimens of plywood. New Zealand Florest Service, FRI Bulletin, n. 83, 1984.

BODIG, J.; JAYNE, B.A. Mechanics of wood and wood composites. New York: Van Nostrand Reinhold, 1993. 711p.

BUNN, E.H. The nature of the resource. New Zealand of Forestry Science, v.26, 

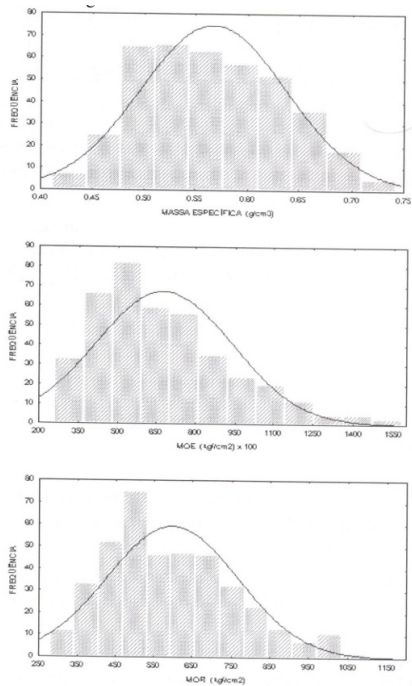

FIGURA 3: Histogramas de distribuição de frequências da massa específica, MOE e MOR dos compensados no sentido paralelo.

FIGURE 3: Histograms of frequency distribution of the specific mass, MOE and MOR of plywoods in the parallel sense.

n.2, p.162-199, 1981.

CAMARGO, F.A. Densidade e lenho tardio de arvores provenientes de desbastes em povoamentos de pinus. Relatório Técnico CAFMA. Piracicaba, 1987.

KOCH, P. Effects of seven variables on properties of Southern pine plywood: part I. For. Prod. J., Medison, v.15, n.9, p.355 - 361, 1965.

LUTZ, J. How growth rate affects properties of softwood veneer. For. Prod. J., Madison, v.14, n.3, p.97-102, 1964.

LUTZ, J. How and log characteristics affecting veneer production. USDA. Forest Service Research Paper, FPL - 150, Madison, 1971.

MUÑIZ, G.I. caracterização e desenvolvimento de modelos para estimar as propriedades e comportamento na secagem da madeira de Pinus elliottii Engelm e Pinus taedae L. Curitiba, 1993, 236p. Tese (Doutorado em Ciência Florestal) - Setor Ciências Agrarias, Universidade Federal do Paraná. 